\title{
Acceptor State Anchoring in Gallium Nitride
}

\author{
Revised 3/2/2020 17:54:00
}

\author{
D. Cameron ${ }^{1 *}$, K.P. O’Donnell ${ }^{1}$, P. R. Edwards ${ }^{1}$, M. Peres ${ }^{2}$, K Lorenz $^{2}$, M. J. Kappers ${ }^{3}$ and M Boćkowski ${ }^{4}$ \\ ${ }^{I}$ SUPA, Department of Physics, University of Strathclyde, Glasgow G4 ONG, Scotland, United Kingdom \\ ${ }^{2}$ IPFN, INESC-MN Instituto Superior Técnico, Universidade de Lisboa, Campus Tecnológico e Nuclear, \\ Estrada Nacional 10, 2695-066 Bobadela LRS, Portugal \\ ${ }^{3}$ Department of Materials Science and Metallurgy, University of Cambridge, 27 Charles Babbage Road, Cambridge CB3 OFS, England, \\ United Kingdom \\ ${ }^{4}$ Institute of High Pressure Physics PAS, Sokolowska 29/37, 01-142 Warsaw, Poland
}

\begin{abstract}
The dual nature of the magnesium acceptor in gallium nitride results in dynamic defect complexes. Europium spectator ions reveal switching between two spectrally unique metastable centres, each corresponding to a particular acceptor state. By ion co-implantation of europium and oxygen into $\mathrm{GaN}(\mathrm{Mg})$, we produce, in addition, an anchored state system. In doing so we create an abundance of previously unidentified stable centres which we denote as "Eu0(Ox)". We introduce a microscopic model for these centres with oxygen substituting for nitrogen in the bridging site.
\end{abstract}

The magnesium $(\mathrm{Mg})$ acceptor is the most common commercially employed dopant for creating effective p-type III-nitrides for optoelectronics. Despite widespread use, the true nature of the magnesium acceptor is much debated and its interplay with other atomic species within the GaN lattice remains mysterious. During the growth of GaN many unintentional dopants will be incorporated, such as oxygen, hydrogen and carbon [1], rendering the interaction of magnesium with many other atomic species inevitable. These dopant species can cause numerous negative effects on the electrical and optical properties of the crystal, but may also be beneficial.

The rare-earth element europium $(\mathrm{Eu})$ is incorporated into $\mathrm{GaN}$ in an attempt to create efficient optoelectronic devices that emit in the red [2]. In GaN, trivalent rare earth $\left(\mathrm{RE}^{3+}\right)$ ions present sharp intra- $4 f$ transition lines. Small changes in the ion's local environment will induce Stark splittings in the RE spectrum. This allows for these ions to be used as spectator ions to examine defect complexes within the crystal.

Together, within a GaN host, europium and magnesium form defect complexes displaying a curious behaviour [3]. At room temperature, high quality $\mathrm{GaN}$ codoped with $\mathrm{Eu}$ and $\mathrm{Mg}$ displays luminescence spectra dominated by a single defect centre (named "Eu0" [4]). Upon cooling $(\approx 20 \mathrm{~K})$ a sample under excitation, one observes a switch in optical dominance, from Eu0 to a more symmetric $\mathrm{Eu} 1(\mathrm{Mg})$ centre. This increase in symmetry is most clearly seen in the ${ }^{5} \mathrm{D}_{0} \rightarrow{ }^{7} \mathrm{~F}_{1}$ transition with the narrowing of the doublet, attributed to a photo-induced migration of the magnesium acceptor. To understand why this migration occurs one must recognise the true character of the magnesium acceptor.

Monemar [5] et al were the first to definitively observe experimentally two distinct acceptor states. Following this Lany and Zunger introduced the concept of metastable shallow transient and deep ground states [6]. The shallow transient state (STS) exists when the acceptor's hole is delocalised, resulting in equal bond lengths to nearest nitrogen neighbours $(2.05 \AA)$. The STS produces an Eu0 centre when present in a complex with a europium ion [7]. When the hole becomes localised at one specific bond, a deep ground state (DGS) is formed, elongating one bond length to $2.23 \AA$. It has been debated as to which particular bond the hole is localised to, with Lany and Zunger's original model suggesting a basal bond and latterly works by Callsen, Lyons and Davies suggesting an axial bond localisation [810]. The particular model by Singh et al, which we extend in this work, favours an axial localization, driving the acceptor away from the interlinking nitrogen ion [7]. This effectively increases the Eu-Mg separation and is the root cause of the symmetry increase observed in the $\mathrm{Eu} 0 \rightarrow \mathrm{Eu} 1(\mathrm{Mg})$ transition.

Switchback from Eu1(Mg) to Eu0 upon warming occurs at higher temperatures $(\approx 100 \mathrm{~K})$ giving a hysteretic defect switching cycle. The behaviour of this metastable system is therefore described as hysteretic photochromic switching (HPS).

Oxygen incorporated during the growth of $\mathrm{GaN}$ is known to influence carrier concentrations, implying that oxygen acts as a shallow donor and likely lies on a nitrogen site [11]. This substitutional oxygen is shown to be singly positively charged and have a very low formation energy, lower than that of a nitrogen vacancy; this gives an explanation as to why oxygen is likely the cause of the unintentional $n$-type conductivity of GaN. Other oxygen sites such as interstitials and gallium substitutions are possible, although these alternative oxygen placements would not be energetically favourable. Calculations have shown that oxygen ions may form complexes with gallium vacancies to reduce Coulomb energy [12]. It is expected that these defects may contribute to broad band yellow luminescence (YL), via shallow donor to deep acceptor transitions $[13,14]$. This case is supported 
further by positron annihilation spectroscopy measurements which show a relation between the negatively charged gallium vacancy concentration and YL intensity [15]. When forming complexes with magnesium the most energetically favourable configuration would be neighbouring substitutional sites with the oxygen sitting on the nitrogen site and magnesium on a gallium site. When in this configuration, ions lie almost directly substitutional with minimal influence on neighbouring atoms in the crystal [16].

Only recently has the role of oxygen in europium doped $\mathrm{GaN}$ been investigated. Experimentally oxygen was found to play a critical role in facilitating europium incorporation during MOVPE growth. [17]. Rutherford backscattering spectrometry measurements showed that with oxygen free growth only $70 \%$ of Eu ions sat on the desired substitutional $\mathrm{Eu}_{\mathrm{Ga}}$ sites, with the rest incorporated as interstitials. From this, it is apparent that an oxygen presence is beneficial for the growth (although for electroluminescent technologies the quantity must be limited, as a portion of these ions will be electrically active). Unfortunately this work was not extended to p-type GaN.

Theoretical works by Massago et al have predicted that co-doping $\mathrm{GaN}$ with europium, magnesium and oxygen may be advantageous for optoelectronic devices $[18,19]$. They predict stable and efficient light-emitting europium centres thanks to acceptor and donor levels improving excitation pathways. Until now, no experimental work has been able to test this prediction. Here we experimentally examine $\mathrm{GaN}: \mathrm{Eu}, \mathrm{Mg}, \mathrm{O}$ using temperature dependent photoluminescence spectroscopy and relate our results to previous work, producing a model of the defect complexes in the system and describing their properties.

For temperature dependent photoluminescence (TDPL) measurements, excitation was preformed using a $355 \mathrm{~nm} \mathrm{CW}$ laser (Cobolt Zouk) with a maximum power of $20 \mathrm{~mW}$ passed through neutral density filter to reduce power down to $2 \mathrm{~mW}$ over a $1.5 \mathrm{~mm}$ spot. The light emitted from the sample was collected into a $0.67 \mathrm{~m}$ spectrometer (McPherson 207) and dispersed over a cooled CCD (Andor Technology). For room temperature measurements excitation was preformed by a $1000 \mathrm{~W}$ xenon arc lamp fed through a monochromator to obtain a $355 \mathrm{~nm}$ beam. The collection system remains the same as for the temperature dependent measurements.

GaN:Mg was grown on sapphire by MOVPE, then ion implanted with $\mathrm{Eu}(300 \mathrm{keV})$ and either $\mathrm{N}(40 \mathrm{keV})$ or $\mathrm{O}$ $(37 \mathrm{keV})$. This resulted in a maximum overlap of doping profiles $60 \mathrm{~nm}$ below the surface. For samples implanted with nitrogen and oxygen using fluences ranging from $1 \times 10^{13} \mathrm{~cm}^{-2}$ to $1 \times 10^{14} \mathrm{~cm}^{-2}$, europium was implanted with $3 \times 10^{13} \mathrm{~cm}^{-2}$. This results in peak concentrations of nitrogen and oxygen $\approx 1.4 \times 10^{18} \mathrm{~cm}^{-3}$ to $1.4 \times 10^{19} \mathrm{~cm}^{-3}$ and a europium concentration of $6 \times 10^{18} \mathrm{~cm}^{-3}$ to match the mean magnesium concentration. Samples implanted with more extreme oxygen fluences of $3 \times 10^{14} \mathrm{~cm}^{-2}\left(4.2 \times 10^{19} \mathrm{~cm}^{-3}\right)$ and $1 \times 10^{15} \mathrm{~cm}^{-2}\left(1.4 \times 10^{20} \mathrm{~cm}^{-3}\right)$ were also prepared, but with higher levels of europium $-1 \times 10^{14} \mathrm{~cm}^{-2}\left(2 \times 10^{19} \mathrm{~cm}^{-3}\right)$ - and magnesium which decreases some broad band luminescence. To repair damage caused by ion implantation, high temperature high pressure (HTHP) annealing was performed at $1400^{\circ} \mathrm{C}$ under $1 \mathrm{GPa}$ of $\mathrm{N}_{2}$ for 30 minutes. This corresponds the optimum annealing conditions for optical activation of Eu. [20]

Room temperature photoluminescence spectra taken from oxygen implanted samples and a nitrogen implanted sample are compared in Figures 1(a) and 1(b). The nitrogen implanted sample displays a classic Eu0 emission spectrum, which can be confirmed by the wavenumber table in ref [21]. The oxygen implanted samples show a superficially similar, but nonetheless distinct emission spectrum. The majority of spectral lines appear only to be slightly shifted, by around $\pm 0.01 \mathrm{~nm}$ on average. The largest disparity occurs in the ${ }^{5} \mathrm{D}_{0} \rightarrow{ }^{7} \mathrm{~F}_{2}$ doublet near $630 \mathrm{~nm}$ where the shift is closer to $1 \mathrm{~nm}$ (Figure 1(b)). This minimal but measurable perturbation of the spectral landscape explains why the existence of these centres has gone hitherto unrecognised. These centres will henceforth be referred to as $\mathrm{Eu0}(\mathrm{Ox})$ due to their spectral similarities with "real" Eu0 sites.
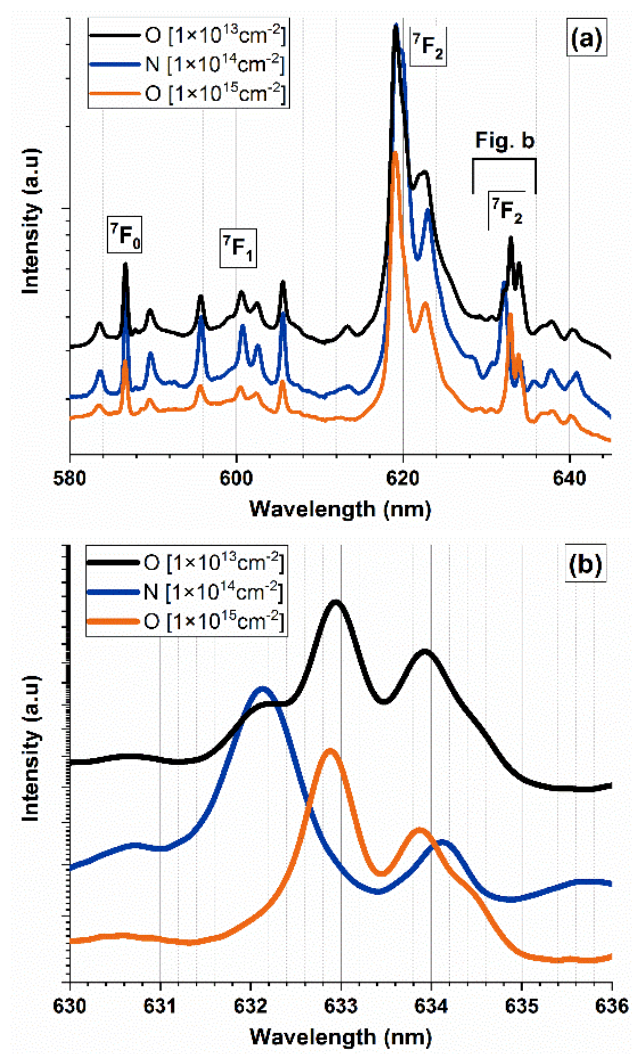

Figure 1: Room temperature PL spectra for three samples. Spectral region (a) includes the entirety of ${ }^{5} D_{0}$ to ${ }^{7} F_{0,1,2}$ transitions and some ${ }^{5} D_{0}$ to ${ }^{7} F_{3}$ transitions $(b)$ Highlights $a^{5} D_{0}$ to ${ }^{7} F_{2}$ doublet which exhibits the largest spectral disparity of nearly $1 \mathrm{~nm}$.

Although not shown here, the presence of substantial populations of $\mathrm{Eu} 0(\mathrm{Ox})$ is accompanied by an increase in competing broad band (peaks at $405 \mathrm{~nm}$ and $570 \mathrm{~nm}$ ) and near 
band edge $(365 \mathrm{~nm})$ luminescence, resulting in a decrease in europium luminescence. This is not unexpected, with excess oxygen shallow donors potentially pairing with deep acceptor gallium vacancies allowing for the shallow-deep transitions required for YL. It should be mentioned that the broad band behaviour of these samples is non-trivial, being affected by dopants, implantation defects and annealing, so will not be discussed in detail here. The presence of these additional broad bands would be detrimental to creating an efficient light emitting device in the red.

The existence of oxygen induced centres is further evidenced by TDPL measurements. The luminescence from each defect centre species should have a characteristic dependence on temperature. The expected TDPL behaviour in the ${ }^{5} \mathrm{D}_{0} \rightarrow{ }^{7} \mathrm{~F}_{0}$ region in oxygen free samples is shown in Figure 2(a). The Eu0 singlet transmission rapidly decreases at low temperatures, as the population evolves and acceptors migrate forming Eu1( $\mathrm{Mg})$ centres. This particular sample has been implanted with nitrogen to explore the possibility that the ballistic ion implantation may be causing the formation of new defect centres. As can be seen in the spectra, there is no obvious indication that this is the case. The nitrogen implanted samples, regardless of implantation fluence, all display Eu0 and Eu1(Mg) emission. This is likely thanks to the post implantation HTHP annealing acting as a quasi-regrowth step and repairing any damage.

An example of the TDPL of a highly oxygen implanted sample is shown in Figure 2(b). Here we observe the coexistence of Eu0 and Eu0(Ox). During cooling under excitation the real Eu0 population will begin migrating into Eu1(Mg) through HPS as expected, while the Eu0(Ox) acceptor state remains anchored.

The highly oxygen implanted samples also display two unidentified emission lines in this region. By virtue of the singlet nature of the ${ }^{5} \mathrm{D}_{0->}>\mathrm{F}_{0}$ transition we expect each centre to display one line or no line regardless of symmetry. This implies that these extra lines correspond to emission from another two centres. Note however, that the intensities of the lines here do not directly reflect the populations of their source centres. This is because this forbidden transition can only occur as a result of J-mixing or Wybourne-Downer mechanisms [22]. These both favour low symmetry sites, requiring a relaxation of the selection rules. A small population of low symmetry centres could therefore "outshine" a large population of highly symmetric centres in this spectral region, meaning these extra lines may be from small populations of low symmetry centres.

Although the fluences used to produce the samples examined in Figures 2(a) and 2(b) differ, similar behaviours occur in samples implanted to a greater and lesser degree.

The temperature dependence of each centre is plotted in Figure (3) to allow for easy comparison. The data here stresses the stability of Eu0(Ox) and its anchored states over prolonged excitation times and various temperature ranges. Even at a temperature of $10 \mathrm{~K}$ the population remains non labile and emission intensity stable.
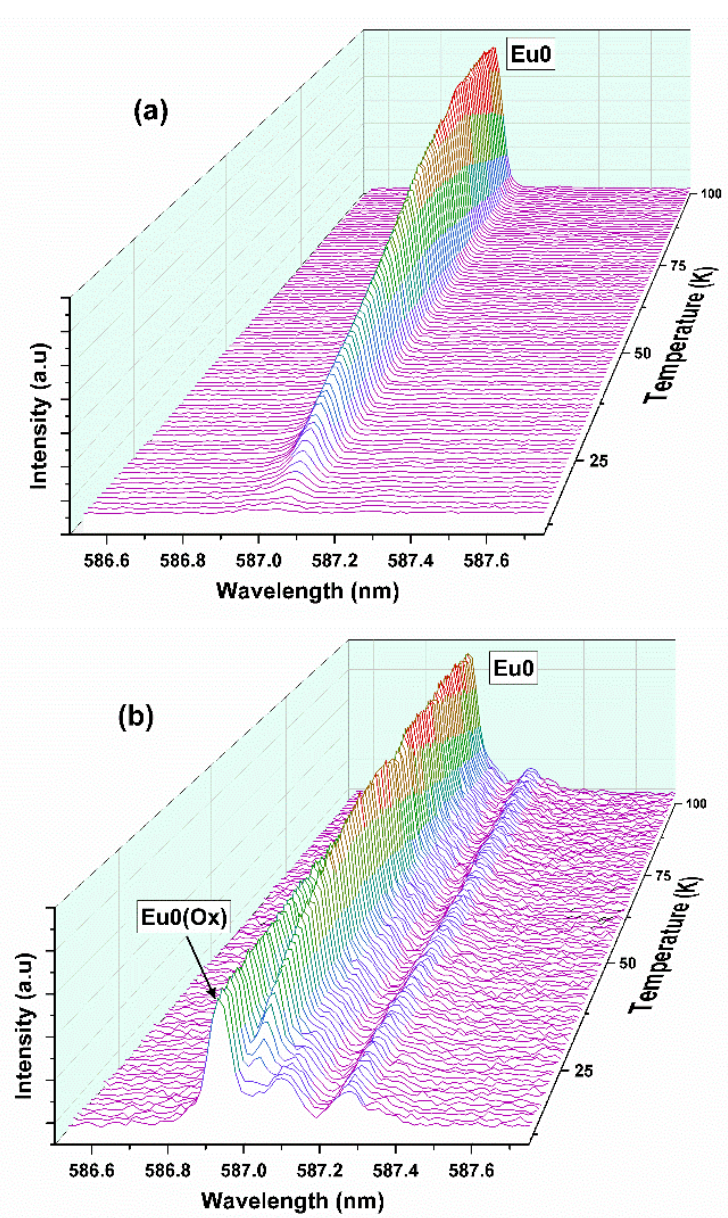

Figure 2: TDPL measurements acquired during cooling from $295 \mathrm{~K}$ to $10 \mathrm{~K}$. (a) Shows the spectra from a nitrogen implanted $\left(10^{13} \mathrm{~cm}^{-2}\right)$ sample displaying the familiar decrease of $\mathrm{EuO}(\mathrm{Ox})$ as population transfer occurs. (b) Shows the spectra from an oxygen implanted $\left(10^{15} \mathrm{~cm}^{-2}\right)$ sample displaying new spectral lines and dependencies in addition to the expected decrease of $E u(O)$. The strongest among these new lines following the decay of EuO is the close lying $\mathrm{EuO}(\mathrm{Ox})$.

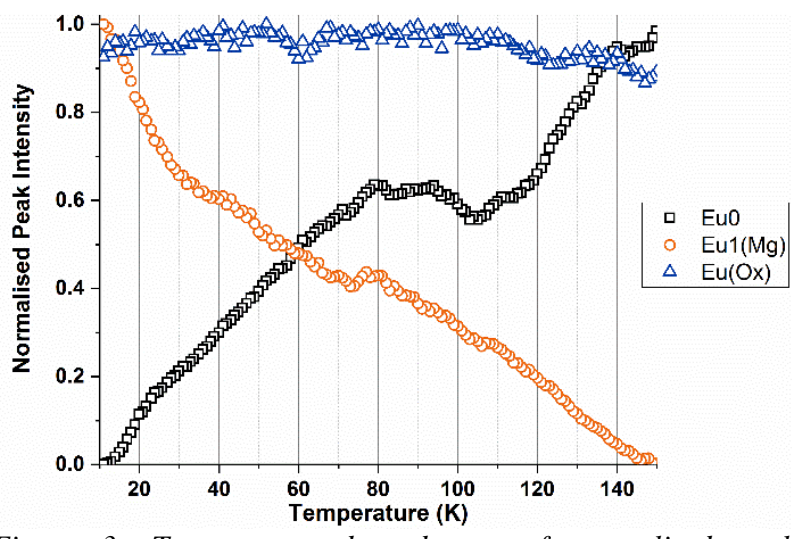

Figure 3: Temperature dependences of normalised peak intensities for the three dominant centres. The data for EuO and Eul(Mg) were extracted from a TDPL cooling run of a nitrogen implanted sample. The EuO(Ox) signal was traced from an oxygen implanted sample. 
In proposing a coherent configuration for these defects, we extend a previous model describing the $\mathrm{Eu0}$ and $\mathrm{Eu} 1(\mathrm{Mg})$ centres. This model, based on dynamic acceptor states and mobile ions, is modified to include the implanted oxygen and formation of the novel $\mathrm{Eu} 0(\mathrm{Ox})$. The accepted structure for the Eu0 and Eu1(Mg) defects as described by Singh et al [7] consists of both centres having similar $\mathrm{Eu}_{\mathrm{Ga}}-\mathrm{N}-\mathrm{Mg}_{\mathrm{Ga}}$ compositions but unique magnesium axial bond lengths. This is due to the transformation in acceptor states from STS to DGS; these centres can be seen in Figures 4(a) and 4(b) respectively.

From energetic considerations the oxygen will likely lie at a nitrogen substitutional site. When neighbouring a magnesium acceptor, these form the closest donor-acceptor pair possible, existing as an isoelectronic complex. For single europium centres containing oxygen and magnesium in $\mathrm{GaN}$ the most favourable configuration is $\mathrm{Eu}_{\mathrm{Ga}}-\mathrm{O}_{\mathrm{N}}-\mathrm{Mg}_{\mathrm{Ga}}$, seen in Figure 4(c). [18]

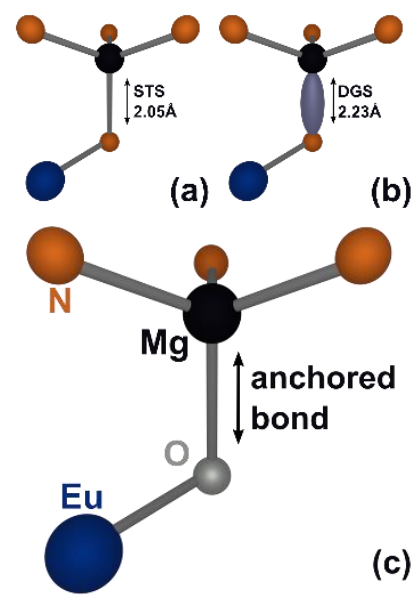

Figure 4: Microscopic models displaying (a) EuO, (b) Eul(Mg) and (c) EuO(Ox); nitrogen in orange, europium in blue, magnesium in black and oxygen in grey. An extended axial magnesium bond length is shown in Eul(Mg), and a strong anchoring bond between magnesium and oxygen is shown in $\mathrm{EuO}(\mathrm{Ox})$.

From this system we can form an explanation as to why $\mathrm{Eu} 0(\mathrm{Ox})$ is state anchored and does not undergo HPS despite its spectral resemblance to $\mathrm{Eu} 0$. In the $\mathrm{Eu} 0(\mathrm{Ox})$ complex, out of the four bonds the magnesium acceptor will make, the $\mathrm{Mg}-\mathrm{O}$ bond will be the strongest. This is caused by a significant increase in ionicity (Pauling contrast +0.4 ) and will force an anisotropic hole distribution. Despite the unusual state, large disturbances of the spectator ion's local crystal environment will be avoided thanks to the acceptor remaining close to the ideal substitutional site and causing minimal external relaxation. A minimal change in electric field would induce a stark splitting congruent with what we observe spectroscopically.

To conclude, we have unveiled a europium magnesium defect with a previously unseen acceptor state, responsible for anchored acceptor states resistant to hysteretic photochromic switching. This novel defect is formed when an oxygen ion substitutes for the bridging nitrogen between the europium spectator and magnesium acceptor. The stability of these centres is attributed to the increase in bond strength between the axial acceptor and bridging ion, causing an anisotropic hole distribution preventing the conventional shallow transient and deep ground states of the acceptor forming.

\section{Acknowledgements}

KPO'D and PRE acknowledge funding from EPSRC, UK (EP/N00275X/1). KL and MP acknowledge funding from FCT Portugal grant PTDC/CTM-CTM/28011/2017, LISBOA-01-0145-FEDER-028011. The data presented in this paper is available at DOI: [add at proof stage]

\section{References}

[1] M. A. Reshchikov, H Morkoç, Journal of applied physics, 97, 061301 (2005); doi:10.1063/1.1868059

[2] A. Nishikawa, T. Kawasaki, N. Furukawa, Y. Terai, and Y. Fujiwara, Appl. Phys. Express 2, 071004 (2009); doi:10.1143/2.071004

[3] K.P. O'Donnell, R. W. Martin, P. R. Edwards, K. Lorenz, E. Alves, M. Boćkowski, AIP Conference Proceedings 1566, 63 (2013); doi:10.1063/1.4848286

[4] K.P. O’Donnell, P.R. Edwards, M. Yamaga, K. Lorenz, M. J. Kappers and M. Boćkowski, Appl. Phys. Lett. 108, 022102 (2016); doi:10.1063/1.4939631

[5] A. Monemar, P. P. Paskov, G. Pozina, C. Hemmingsson, J. P. Bergman, T. Kawashima, H. Amano, I. Akasaki, T. Paskova, S. Figge, D. Hommel, and A. Usui, Phys. Rev. Lett. 102, 235501 (2009); doi:10.1103/PhysRevLett.102.235501

[6] S. Lany \& A. Zunger, Appl. Phys. Lett.96, 142114 (2010); doi:10.1063/1.3383236

[7] A. K. Singh, K. P. O'Donnell, P. R. Edwards, K. Lorenz, M. J. Kappers, M. Bokowski, Sci. Rep. 7 article 41982 (2017); doi:10.1038/srep41982

[8] G. Callsen, M. R. Wagner, T. Kure, J. S. Reparaz, M. Bugler, J. Brunnmeier, C. Nenstiel, A. Hoffmann, M. Hoffmann, J. Tweedie, Z. Bryan, S. Aygun, R. Kirste, R. Collazo, and Z. Sitar, Phys. Rev. B 86, 075207 (2012); doi:10.1103/PhysRevB.86.075207

[9] J. L. Lyons, A Janotti, C. Van de Walle, PRL 108, 156403 (2012); doi:PhysRevLett.108.156403

[10] J. J. Davies, Phys. Rev. B 87, 235208 (2013);

doi:PhysRevB.87.235208

[11] T. Mattila, R. M. Nieminen Phys. Rev. B 54, 16676 (1996); doi:PhysRevB.54.16676

[12] T. Mattila and R. M. Nieminen, Phys. Rev. B 55, 9571 (1997); doi:PhysRevB.55.9571

[13] M. A. Reshchikov, H. Morkoç, S. S. Park, and K. Y. Lee, Appl. Phys. Lett. 81, 4970 (2002); doi: 10.1063/1.1531227

[14] J. Lyons, A Alkauskas, A Janottti and C.G. Van de Walle, Phys. Status. Solidi B 252, No. 5, 900-908 (2015); doi:10.1002/pssb.201552062 [15] K Saarinen, Phys Rev Lett 79, 3030 (1997); doi:PhysRevLett.79.3030 [16] I. Gorczyca, A. Svane, and N. E. Christensen, Phys. Rev. B, 61, 7494 (2000); doi: 10.1103/PhysRevB.61.7494

[17] B. Mitchell, D. Timmerman, J. Poplawsky, W. Zhu, D. Lee, R.Wakamatsu, J. Takatsu, M. Matsuda, W. Guo, K. Lorenz, E. Alves, A.Koizumi, V. Dierolf, Y. Fujiwara, Sci. Rep. 6, (2016); doi: $10.1038 /$ srep 18808

[18] A Masago, T Fukushima1, K Sato and H Katayama-Yoshida, Appl. Phys. Express, 7, 121002, (2014); doi:10.7567/APEX.7.071005

[19] A. Masago, M. Uemoto, T. Fukushima, K. Sato, and H. KatayamaYoshida, Japanese Journal of Applied Physics 56, 021001 (2017); doi:10.7567/JJAP.56.021001 


\section{APPLIED PHYSICS LETTERS}

[20] K. Lorenz, S. M. C. Miranda, E. Alves, I. S. Roqan, K. P. O'Donnell, and M. Boćkowski, "High pressure annealing of Europium implanted

GaN", Proc. of SPIE Vol. 8262, 82620C (2012); doi: 10.1117/12.90681

[21] A. K Singh, K.P. O’Donnell, P. R. Edwards, D. Cameron, K. Lorenz, M. J. Kappers, M. Boćkowski, M. Yamaga and R Prakash, Appl. Phys.

Lett. 111, 241105 (2017); doi:10.1063/1.5001143

[22] P. Tanner, Chem. Soc. Rev, 42, 5090. (2013);

doi:10.1039/C3CS60033E 